\title{
Envenenamento por serpentes do gênero Bothrops no Estado da Bahia: aspectos epidemiológicos e clínicos
}

\author{
Envenomation by Bothrops in the State of Bahia: \\ epidemiological and clinical aspects
}

\author{
Yukari Figueroa Mise', Rejâne Maria Lira-da-Silva ${ }^{1}$ e Fernando Martins Carvalho ${ }^{2}$
}

\begin{abstract}
RESUMO
Descreve-se características clínico-epidemiológicas de 655 casos de acidentes botrópicos atendidos e/ou notificados ao Centro de Informações Antiveneno da Bahia, no Estado da Bahia, em 2001. A incidência anual no Estado foi de 5,0 acidentes/100.000 habitantes e a letalidade, 1\%. A incidência foi máxima na microrregião Litoral Norte (21,9/100.000 habitantes) e no município de Itanagra (92,9/100.000 habitantes). Os acidentes foram predominantemente diurnos, acometendo membros inferiores, em homens, de 11-30 anos, trabalbadores, no ambiente rural e no período chuvoso. Atendimento médico após 13 horas da ocorrência da picada ocorreu em 19\% dos casos. Predominaram os quadros clínicos moderados (47,8\%), seguidos dos graves (23,6\%). As manifestações clínicas locais e sistêmicas seguiram o padrão nacional para as serpentes do gênero Bothrops. Sintomatologias neurológicas, não usualmente atribuídas ao acidente botrópico, foram registrados em alguns casos. A soroterapia empregada (7,7 ampolas/paciente) foi compatível com o fato da maioria de casos serem moderados. Outros tipos de soro que não o univalente foram utilizados em 15 (2,3\%) pacientes.
\end{abstract}

Palavras-chaves: Bothrops Jararaca. Picadas de cobra. Epidemiologia. Antivenenos.

\begin{abstract}
This study describes the clinical and epidemiological characteristics of 655 cases of Bothrops snakebites that were attended by and/or notified to the Bahia Antivenin Information Center, State of Bahia, Brazil, in 2001. The annual incidence in the State was 5.0 cases/100,000 inhabitants and lethality was 1\%. The incidence was greatest in the North Coast microregion (21.9/100,000 inhabitants) and the municipality of Itanagra (92.9/100,000 inhabitants). The snakebites occurred predominantly during the day, affecting the lower limbs of men aged 11-30 years who worked in rural areas during the rainy period. Medical care was obtained more than 13 hours after the snakebite in $19 \%$ of the cases. Moderate (47.8\%) and severe (23.6\%) clinical presentations prevailed. The local and systemic clinical manifestations followed the usual pattern for Bothrops snakebites in Brazil. However, a few cases presented neurological manifestations, which are not usually attributed to Bothrops snakebites. The antivenin therapy used (7.7 blisters/patient) reflected the fact that the majority of the cases were moderate. Antivenins other than the univalent type were used in $2.3 \%$ of the cases.
\end{abstract}

Key-words: Bothrops jararaca. Snakebites. Epidemiology. Antivenins.

Os acidentes ofídicos são um importante problema de saúde pública, especialmente em regiões tropicais do mundo. A obrigatoriedade da notificação, a partir de 1988/89, permitiu um maior conhecimento sobre os envenenamentos ofídicos no Brasil. Apesar disso, as características clínico-epidemiológicas e a real magnitude dos acidentes ofídicos no País ainda são precariamente conhecidas nas regiões Norte e Nordeste, devido à subnotificação ou da informação colhida com omissões ${ }^{15}$. Dentre os acidentes por serpentes, 0 acidente botrópico destaca-se pela sua elevada incidência ${ }^{824}$.

0 presente trabalho descreve características clínicas e epidemiológicas de envenenamentos botrópicos ocorridos no Estado da Bahia, em 2001.

\footnotetext{
1. Departamento de Zoologia, Instituto de Biologia, Universidade Federal da Bahia, Salvador, BA. 2. Departamento de Medicina Preventiva e Social, Faculdade de Medicina, Universidade Federal da Bahia, Salvador, BA.

Órgãos financiadores: PIBIC/UFBA - CNPq, PRODOC/UFBA - FAPESB (Fundação de Amparo á Pesquisa do Estado da Bahia) e FUNASA/MS (Fundação Nacional de Saúde, Ministério da Saúde).

Endereço para correspondência: Prof ${ }^{a}$ Rejâne Maria Lira-da-Silva. Depto de Zoologia/IB/UFBA. Núcleo Regional de Ofiologia e Animais Peçonhentos da Bahia, Campus Universitário de Ondina, Ondina, 40170-210 Salvador, BA.

Tel: $55713283-6564$

e-mail: yukari@ufba.br; rejane@ufba.br; fmc@ufba.br

Recebido para publicação em: 23/07/2007

Aceito em: 23/08/2007
} 


\section{MATERIAL E MÉTODOS}

Realizou-se um estudo descritivo retrospectivo das características clínico-epidemiológicas dos casos de acidentes botrópicos atendidos e/ou notificados ao Centro de Informações Antiveneno da Bahia da Secretaria de Saúde da Bahia (CIAVE/SESAB), no ano de 2001. As características clínicas e epidemiológicas analisadas neste artigo provieram das informações constantes nas Fichas Individuais dos casos, padronizadas pelo Programa Nacional de Ofidismo e Animais Peçonhentos. As Fichas eram preenchidas pelos médicos do CIAVE e/ou da emergência do Hospital Central Roberto Santos (HCRS), a partir dos prontuários dos pacientes.

A área de pesquisa compreendeu o Estado da Bahia, com área de $567.295 \mathrm{~km}^{2}$, com 417 municípios e população de 13.214 .114 habitantes. Foram calculadas as medidas de incidência e letalidade para a Bahia, referentes ao ano de 2001. De acordo com a configuração geopolítica, o Estado está dividido em 15 subzonas $^{30}$. Como denominadores das taxas de incidência, usou-se os dados demográficos populacionais disponíveis no IBGE9. 0 Risco foi definido como a probabilidade de um indivíduo ou grupo de indivíduos sofrerem 0 acidente ofídico em relação à respectiva população de base, no ano de 2001.

Para processamento e análise dos dados, utilizou-se 0 programa SPSS $^{\circledast}$ versão 11.0 (Statistical Package for the Social Sciences) $)^{31}$.

A identificação das serpentes causadoras dos acidentes foi feita pelo Núcleo Regional de Ofiologia e Animais Peçonhentos, Instituto de Biologia da Universidade Federal da Bahia (NOAP/ UFBA).

\section{RESULTADOS}

Aspectos epidemiológicos. Em 2001, o CIAVE-BA atendeu e/ou notificou 740 acidentes ofídicos; destes, 665 (89,9\%) foram acidentes botrópicos perfazendo uma incidência de 5,0 acidentes botrópicos/100.000 habitantes. Apenas $11(1,7 \%)$ casos foram confirmados com a identificação da serpente causadora do acidente, sendo todos exemplares da espécie Bothrops leucurus.

0 Litoral Norte foi a microrregião que apresentou maior incidência de acidentes botrópicos (21,9 casos/100.000 habitantes) e o município mais afetado foi Itanagra (92,9 casos/100.000 habitantes). Em Salvador, os bairros mais atingidos foram Pirajá e Fazenda Grande do Retiro, cada qual contribuindo com $16 \%$ dos casos ocorridos nesta capital. Os acidentes botrópicos ocorreram mais no período chuvoso de março-agosto $(62,4 \%)$ que no período menos chuvoso de setembro-fevereiro $(37,6 \%)$.

Na casuística predominou o sexo masculino $(76,7 \%)$ e pacientes com faixa etária de 11 a 30 anos (46,5\%). Informação sobre a ocupação só existia para 188 dos 665 casos: 92 eram menores de 10 anos, 4 eram aposentados ou desempregados e para 381 não havia essa informação. A ocupação mais frequientemente registrada foi a de trabalhador rural (110 casos ou $16,5 \%$ ). Elevada proporção (542 casos ou $81,5 \%$ ) dos acidentes ocorreu em ambiente rural. 0 trabalho habitual foi a atividade mais freqüentemente mencionada no momento da picada $(66,7 \%)$, seguida pelo lazer $(21,1 \%)$.

A maioria (413 casos ou 62,1\%) dos pacientes chegou ao hospital nas seis primeiras horas após a picada. Por outro lado, $19 \%$ dos pacientes chegaram ao atendimento médico com mais de 13 horas decorridas após a picada. Os acidentes botrópicos acometeram principalmente os pés ou as pernas $(75,2 \%)$ e ocorreram no período diurno (76,7\%). Dentre os 665 acidentes botrópicos registrados no ano de 2001, ocorreram sete óbitos, o que correspondeu à letalidade de $1 \%$.

Aspectos clínicos. Os acidentes foram classificados como leves $(19,1 \%)$, moderados $(47,8 \%)$ ou graves $(23,6 \%)$.

As manifestações no local da picada mais verificadas nestes pacientes foram: edema $(90,7 \%)$, sangramento $(7,8 \%)$ e equimose (6,3\%) (Tabela 1). As manifestações sistêmicas relacionadas às alterações no tempo de coagulação foram observadas em 66,5\%

Tabela 1 - Manifestações no local da picada de 665 acidentes botrópicos do ano de 2001 na Bahia, Brasil.

\begin{tabular}{lcc}
\hline Manifestações no local da picada & Número & Percentual \\
\hline Edema & 603 & 90,7 \\
Sangramento & 52 & 7,8 \\
Equimose & 42 & 6,3 \\
Bolha & 27 & 4,1 \\
\hline
\end{tabular}

nos 496 pacientes em que se pesquisou estes distúrbios. Cefaléia (5,3\%), gengivorragia $(5,1 \%)$ vômito, hematúria e alteração na cor da urina (todos com 3,2\%) também foram manifestações verificadas (Tabela 2).

Soroterapia. Dentre os 665 pacientes com diagnóstico de acidente botrópico, 621 (93,4\%) receberam soro antibotrópico, 7 (1\%) receberam soro antibotrópico-laquético, $6(0,9 \%)$ receberam soro antibotrópico-crotálico, $2(0,3 \%)$ receberam soro anticrotálico, e $29(4,4 \%)$ não receberam qualquer tipo de soro. Dentre os 636 pacientes que receberam soroterapia antiofídica, a média de ampolas na primeira dose foi de 8,1 ampolas/paciente. Houve uma segunda dose para 59 (9,3\%) destes pacientes, que variou de 1 a 4 ampolas $(62,7 \%$, de 59 casos), 5 a 8 ampolas (23,7\%) de 9 a 12 ampolas (13,6\%). Dentre os 621 pacientes que receberam o soro antibotrópico, 57 (9,1\%) apresentaram reações imediatas: febre (28 casos), palidez (6 casos), náuseas/vômitos, taquicardia e hipotensão/ choque ( 5 casos cada), taquipnéia/dispnéia e broncoespasmo (1 caso cada).

0 torniquete foi utilizado como intervenção inicial em $59(8,9 \%)$ casos. Onze (1,7\%) pacientes relataram o uso de recursos da medicina popular previamente ao atendimento médico. 
Tabela 2 - Manifestações sistêmicos de 665 acidentes botrópicos do ano de 2001 na Babia, Brasil.

\begin{tabular}{|c|c|c|c|c|c|}
\hline Manifestações sistêmicas & $\mathrm{n}^{\mathrm{o}}$ & $\%$ & Manifestações sistêmicas & $\mathrm{n}^{\mathrm{o}}$ & $\%$ \\
\hline Coagulação sangüínea alterada* & 330 & 66,5 & Torpor & 4 & 0,6 \\
\hline Cefaléia & 35 & 5,3 & Hemoptise & 4 & 0,6 \\
\hline Gengivorragia & 34 & 5,1 & Dor abdominal & 3 & 0,5 \\
\hline Vômito & 21 & 3,2 & Tosse & 3 & 0,5 \\
\hline Hematúria & 21 & 3,2 & Hipotensão & 3 & 0,5 \\
\hline Cor da urina alterada & 21 & 3,2 & Hipotermia & 2 & 0,3 \\
\hline Tonturas & 12 & 1,8 & Coma & 2 & 0,3 \\
\hline Visão turva & 12 & 1,8 & Agitação & 2 & 0,3 \\
\hline Sonolência & 11 & 1,7 & Convulsão & 2 & 0,3 \\
\hline Hipertermia & 9 & 1,4 & Midríase & 2 & 0,3 \\
\hline Cianose & 9 & $\begin{array}{l}1,4 \\
1,2\end{array}$ & Púrpura & 2 & 0,3 \\
\hline Sudorese & 8 & 1,2 & Choque & 1 & 0,2 \\
\hline Oligúria & 8 & 1,2 & Bradicardia & 1 & 0,2 \\
\hline Palidez & 7 & 1,1 & Taquicardia & 1 & 0,2 \\
\hline Hipertensão & 6 & 0,9 & Diarréia & 1 & 0,2 \\
\hline Oligúria & 6 & 0,9 & Tremores & 1 & 0,2 \\
\hline Náusea & 5 & 0,8 & Ptose palpebral & 1 & 0,2 \\
\hline Epistaxis & 5 & 0,8 & Reflexos alterados & 1 & 0,2 \\
\hline Hematêmese & 5 & 0,8 & Bradipnéia & 1 & 0,2 \\
\hline Mialgia & 4 & 0,6 & Estertores & 1 & 0,2 \\
\hline
\end{tabular}

* Percentual calculado em relação ao total dos 496 pacientes com avaliação do tempo de coagulação.

\section{DISCUSSÃO}

A incidência de acidentes ofídicos na Bahia (5,0 acidentes/ 100.000 hab) é relativamente baixa quando comparada com a média nacional (13,9 acidentes/100.000 hab) e semelhante à média para o Nordeste (7,6 acidentes/100.000 hab) em $1993^{15}$. Esse dado pode estar relacionado à subnotificação do ofidismo no estado, uma vez que as regiões Sul e Sudeste contam com melhor organização dos serviços de saúde e sistemas de informaçã $0^{15}$. Destacou-se o município de Itanagra que possui pecuária desenvolvida e agricultura diversificada. 0 município explora a agricultura de coco e a pecuária, com criação de bovinos e eqüinos, sendo o trabalho agropecuário realizado sem mecanizaçã $0^{13}$. Esse modo de produção pode expor o trabalhador rural a um maior risco de se acidentar. Os bairros de Pirajá e Fazenda Grande do Retiro, em Salvador, caracterizam-se pelo crescimento desordenado, com vestígios de Mata Atlântica e alta densidade populacional, além de saneamento básico precário ou ausente ${ }^{30}$. Estas características propiciam o acúmulo de lixo, entulho e atraem roedores, gerando abrigo e alimento para as serpentes, o que favorece, conseqüentemente, a sua proliferaçã $0^{13}$.

0 hábito de levar a serpente agressora ao serviço de saúde (1,7\%) ainda é uma medida incipiente na Bahia, quando comparada à frequiência de $11,8 \%$, relatada no Estado do Acre ${ }^{17}$. Essa medida é de extrema importância para a identificação do envenenamento e indicar a aplicação de soro antiofídico. Rotineiramente, o CIAVE notifica e encaminha a serpente agressora para o Setor de Biologia do serviço, para a devida identificação. A observação de que a maioria dos acidentes botrópicos acometeu homens na faixa etária dos 11 aos 30 anos e trabalhadores rurais pode ser devido provavelmente à inserção de crianças no trabalho rural, já que houve uma predominância do trabalho habitual como atividade efetivamente mencionada no momento da picada $(66,7 \%)$, seguido por lazer $(21,1 \%)$, similar ao relatado para 0 Brasil e outros países sul-americanos ${ }^{131617}$.

A incidência dos acidentes botrópicos foi mais elevada no período chuvoso. No Brasil, os acidentes ofídicos em geral predominam de janeiro a abril, associados ao aumento da temperatura e pluviosidade, sobretudo nas regiões Sul e Sudeste ${ }^{15}$. $\mathrm{Na}$ Bahia, os acidentes botrópicos registrados predominaram nos meses de março a agosto, devido provavelmente à inundação dos abrigos das jararacas que as obriga a buscar outros esconderijos, tornando-as mais susceptíveis a serem vistas e capturadas por transeuntes ${ }^{17} 18$. Este achado sazonal diferiu daquele relatado para o Rio Grande do $\mathrm{Sul}^{28}$, provavelmente porque na Bahia e em estados da Região Norte não há queda acentuada de temperatura no outono e inverno.

0 tempo entre a picada e o atendimento médico tem grande importância para o prognóstico do acidente ${ }^{11}$. 0 fato de $19 \%$ dos pacientes chegarem ao atendimento médico com mais de 13 horas, indica a desinformação e a precariedade do sistema de atendimento médico, apesar da pequena proporção de pacientes $(1,7 \%)$ ter previamente adotado práticas da medicina popular.

Uma segunda dose foi ministrada em 8,9\% dos pacientes, o que é uma melhor proporção quando comparada com aquela relatada para o Estado do Acre $(16 \%)^{17}$. 0 ideal é que a dose inicial seja suficiente para neutralizar a peçonha inoculada, sem que seja necessária uma dose complementar. 0 soro antibotrópico, assim como os demais soros antiofídicos, deve ser aplicado o mais precocemente possível, pois, evidentemente, só possui ação sobre 0 veneno circulante, não agindo sobre tecidos já lesados ${ }^{32}$.

A predominância de acidentes ofídicos acometendo membros inferiores $(75,2 \%)$ reflete o hábito terrícola da maioria das espécies do gênero Bothrops. Essa situação, observada para a Bahia $^{2} 1314232526$ e para outras regiões do Brasil ${ }^{811} 1921$, denota a não utilização de equipamentos de proteção, em especial nas zonas de agricultura não mecanizada.

Embora as jararacas sejam serpentes noturnas, 76,7\% dos acidentes ocorreram durante o período diurno, possivelmente porque a atividade laboral favorece a possibilidade de contato do homem com a serpente.

Registrou-se o uso de soro anticrotálico em dois pacientes. A administração de soro inadequado pode dever-se a erro grosseiro de diagnóstico, a quadros iniciais que podem simular outro envenenamento ou à falta de soro específico, sendo difícil discutir este assunto. Apesar disso, o uso inespecífico da soroterapia éinjustificado, uma vez que a imunidade cruzada, conferida por diversos tipos de soro, é muito baixa ${ }^{5}$. A aplicação de soro inadequado também foi observada no Estado do Acre ${ }^{17}$, o que sugere a necessidade de treinamento e/ou capacitação para a equipe médica, principalmente sobre a identificação de serpentes e aspectos sintomatológicos, diagnósticos e terapêuticos do ofidismo. 
A média de 7,7 ampolas/paciente encontrada neste estudo pode ser considerada alta, segundo parâmetros nacionais $(5,6$ ampolas/paciente), porém compatível com a obtida para a Região Metropolitana de Salvador ${ }^{13} \mathrm{e}$ com o fato da maioria dos casos terem sido classificados como moderados (47,8\%). Embora o Ministério da Saúde recomende uma dosagem padronizada para o tratamento do envenenamento botrópico, existem variações na média de ampolas utilizadas nas Unidades Federadas: Rio Grande do Norte (3,3 ampolas/paciente), Santa Catarina e Espírito Santo (4,0 ampolas/paciente), Pernambuco (7,3 ampolas/paciente) e Distrito Federal $(8,3 \text { ampolas/paciente })^{15}$. Esta variação provavelmente está associada à realidade de cada região do país e pelos agentes etiológicos envolvidos. Estes fatos sugerem a necessidade, apontada desde $1997^{10}$ de reavaliação da atual recomendaçã $0^{15}$ do número padronizado de ampolas de soro antiofídico a serem utilizadas num tratamento.

As reações precoces à soroterapia são motivos de preocupação, devido à sua potencial gravidade. Existem poucos trabalhos na literatura especializada referentes às reações à soroterapia ${ }^{3}$ apesar do monitoramento do paciente durante a infusão do soro antiofídico ser indispensável para se observar a ocorrência de anafilaxia ${ }^{32}$. Foram observadas reações imediatas à soroterapia em 8,9\% dos pacientes estudados. Essa proporção é baixa, quando comparada à relatada para 0 Estado do Acre ${ }^{17}$, Ribeirão Preto ${ }^{3} \mathrm{e}$ muito abaixo daquela relatada para Salvador, Bahia $(67,5 \%)^{12}$. A baixa proporção de reações ao soro antibotrópico encontrada nesse trabalho pode estar relacionada à falta de observação da equipe médica em relação a determinados sintomas de reações ao soro.

Contra-indicado no envenenamento botrópico ${ }^{15}$, o uso de torniquete foi pouco registrado $(8,9 \%)$, entretanto, 0 preenchimento das informações costuma ser falho e quando o dado é obtido prospectivamente ${ }^{424}$, a percentagem é muito maior do que quando obtida, no mesmo centro de atendimento, porém com base em prontuários ${ }^{1923}$.

Foram verificadas lesões locais como edema $(90,7 \%)$, sangramento $(7,8 \%)$ e equimose $(6,3 \%)$, concordando com o perfil clínico dos acidentes botrópicos na Bahia ${ }^{202526}$ e no Brasil ${ }^{112227}$. Lesões locais estão associadas à ação proteolítica, freqüente no veneno botrópic $0^{9}$. As frequiências de manifestações sistêmicas gerais são semelhantes às descritas para acidentes botrópicos ocorridos na Região Metropolitana de Salvador ${ }^{13}$ e na Bahia ${ }^{2526}$. A menor frequiência de distúrbios digestivos e cardiovasculares também concorda com dados para essas regiões ${ }^{132526}$.

A proporção de casos com gengivorragia $(5,1 \%)$ foi baixa quando comparada com dados do Estado do Acre $(15,3 \%)^{17}$, e equivalente à proporção relatada para Salvador, Bahia $(5,3 \%)^{13}$. Essa diferença provavelmente está relacionada com a variabilidade do veneno entre espécies do mesmo gênero, pois, no Acre, a Bothrops atrox é o principal agente agressor ${ }^{17}$.

As alterações no tempo de coagulação sangüínea observadas nesse trabalho concordam com dados da literatura ${ }^{12123}$. 0 tempo de coagulação sangüínea não possui valor como critério de gravidade, mas é usado como parâmetro na evolução do quadro clínico $^{24}$.
Apesar da baixa frequiência de sintomas urinários observados, é importante salientar que a falência renal é a principal causa de óbito pelo envenenamento botrópico ${ }^{4}$. Esse dado se aproxima dos dados obtidos para a Região Metropolitana de Salvador ${ }^{13}$.

Sintomas neurológicos (principalmente cefaléia) e respiratórios (bradipnéia) ocorreram em proporções semelhantes às relatadas para a Bahia ${ }^{25} 26$ e podem estar relacionados à ação neurotóxica pré-sináptica do veneno de Bothrops leucurus observada in vitro sobre a preparação nervo-frênico diafragma de camundongo ${ }^{12}$. Essa peculiaridade do veneno da Bothrops leucurus sugere que os agentes de saúde devem estar aptos a executar a terapêutica adequada aos sintomas sistêmicos, raros nos acidentes botrópicos no país, porém presentes nos acidentes para o Estado.

A letalidade de $1 \%$ foi alta, se comparada com a média nacional $(0,45 \%)$ e nordestina $(0,81 \%)$ nos anos de 1990 a $1993^{15}$, respectivamente; mas se equipara à média do Estado da Bahia, nos últimos quinze anos. Embora a letalidade na Bahia tenha decrescido nos últimos anos $\left(2,6 \% \mathrm{em} 1985^{25} ; 1,3 \% \mathrm{em}\right.$ $1986^{26} ; 1,1 \%$ em $1991 / 1992^{2} ; 0,5 \%$ em $1995^{29}$ ), esteve sempre acima da média nacional.

A predominância de acidentes moderados $(47,8 \%)$ difere de informação anteriormente relatada para a Região Metropolitana de Salvador, Bahia ${ }^{13}$, quando predominaram os casos leves. Esta discrepância pode dever-se a equívocos no enquadramento do estadiamento do paciente, tais como considerar a alteração no tempo de coagulação como indicativo de gravidade, o que é um procedimento incorreto $0^{23}$.

Diversos fatores podem estar associados à baixa qualidade das anotações nos prontuários médicos. Dentre esses, a dinâmica de atendimento na Unidade de Saúde; o elevado fluxo de pacientes, tanto para acidentes ofídicos quanto para outros problemas de caráter médico; 0 o costume de não se fazerem as devidas anotações em prontuário, por causa da "falta de tempo" dos componentes da equipe de saúde ${ }^{17}$. É de extrema importância que profissionais de saúde estejam capacitados para o manejo epidemiológico, clínico e terapêutico dos acidentes botrópicos, mas infelizmente apenas 56\% das escolas médicas do País preparam seus futuros profissionais para 0 atendimento aos acidentados por animais peçonhentos 7 .

\section{AGRADECIMENTOS}

Os autores agradecem à Dra. Dayse Schwab Rodrigues, Coordenadora do Centro de Informações Antiveneno da Bahia da Secretaria de Saúde da Bahia (CIAVE/SESAB) por ter disponibilizado a consulta às fichas de atendimento de pacientes.

\section{REFERÊNCIAS}

1. Acosta AR, Uzcategui W, Azuaje R, Aguilar I, Girón ME. Análisis clínico y epidemiológico de los accidentes por mordeduras de serpientes del gênero Bothrops en Venezuela. Revista Cubana de Medicina Tropical 52:90-94, 2000.

2. Carmo ICR. Contribuição para o estudo epidemiológico de acidentes ofídicos no Estado da Bahia no período de 1991-1992. Monografia de Bacharelado em Ciências Biológicas, Universidade Federal da Bahia. Salvador, 1994. 
3. Cupo P, Azevedo-Marques MM, Menezes JB, Hering SE. Reações de hipersensibilidade imediatas após uso intravenoso de soros antivenenos: valor prognóstico dos testes de sensibilidade intradérmicos. Revista do Instituto de Medicina Tropical de São Paulo 33:115-122, 1991.

4. Da-Silva CJ, Jorge MT, Ribeiro LA. Epidemiology of snakebite in a central region of Brazil. Toxicon 41:251-255, 2003.

5. Dias-da-Silva W, Guidolin R, Raw I, Higashi HG, Caricati CP, Morais JF, Lima MLSR, Yamaguchi IK, Nishikawa AK, Stephano MA, Marcelino JR, Pinto JR, Santos MJ. Cross-reactivity of horse monovalent antivenoms to venom of ten Bothrops species. Memórias do Instituto Butantan 51:153-168, 1989.

6. Fan HW, Cardoso JL. Clinical toxicology of snakebites in South America. In: Meier J, White J (eds) Handbook of clinical toxicology of animals venoms and poisons. CRC Press, Boca Raton, p. 667-688, 1995.

7. Ferreira Junior JR, Barraviera, B. Artrópodes de Importância Médica. $1^{\mathrm{a}}$ edição Editora de Publicações Biomédicas Ltdd ${ }^{a}$, Rio de Janeiro, 47 p, 2002.

8. França FOS; Málaque CMS. Acidente botrópico. In: Cardoso JLC, França FOS, Wen FH, Málaque CMS, Haddad V (eds) Animais peçonhentos no Brasil. Biologia, clínica e terapêutica dos acidentes. 1ª edição. Sarvier, São Paulo, 468 p, 2003.

9. Instituto Brasileiro de Geografia e Estatística. Censo Demográfico Brasileiro. Acessível em http://www.ibge.gov.br. Acesso em 12/03/2004, 2000.

10. Jorge, MT, Ribeiro, LA. Dose de soro (antiveneno) no tratamento do envenenamento por serpentes peçonhentas do gênero Bothrops. Revista da Associação Médica Brasileira 43: 74-76, 1997.

11. Kouyoumdjian JA, Polizelli C, Lobo SMA, Guimarães SM. Acidentes ofídicos causados por Bothrops moojeni na região de São José do Rio Preto, São Paulo. Arquivos Brasileiros de Medicina 64:167-171, 1990.

12. Lira-da-Silva RM. Estudo farmacológico do veneno de Bothrops leucurus (Serpentes; Viperidae). Tese de Doutorado, Universidade de São Paulo, São Paulo, 2001.

13. Lira-da-Silva RM, Carvalho, FM. Epidemiological clinical study of envenoming by Bothrops leucurus Wagler, 1824 (Serpentes; Viperidae) in the metropolitan area of Salvador, Bahia, Brazil. The Journal of Venomous Animals and Toxins 4: 4-5, 1998.

14. Lira-da-Silva RM, Lima RA, Nunes TB. Envenomation caused by Botbrops leucurus Wagler, 1924 (Serpentes; Viperidae) in the metropolitan region of Salvador, Bahia. Revista da Sociedade Brasileira de Medicina Tropical 27 (supl): 124-124, 1994.

15. Ministério da Saúde. Manual de diagnóstico e tratamento de acidentes ofídicos. Fundação Nacional de Saúde, Coordenação de Controle de Zoonoses e animais peçonhentos, Ministério da Saúde, Brasília, 1999.

16. Morandi N, Williams J. Snakebite injuries: contributing factors and intentionality of exposure. Wilderness \& Environmental Medicine 8:152-155, 1997.

17. Moreno E, Queiroz-Andrade M, Lira-da-Silva RM, Tavares-Neto J. Características clínicoepidemiológicas dos acidentes ofídicos em Rio Branco, Acre. Revista da Sociedade Brasileira de Medicina Tropical 38:15-21, 2005.
18. Nascimento SP. Aspectos epidemiológicos dos acidentes ofídicos ocorridos no Estado de Roraima, Brasil, entre 1992 e 1998. Cadernos de Saúde Pública 16:271-276, 2000 .

19. Nishioka SA, Silveira PVP. A clinical and epidemiological study of 292 cases of lance-headed viper bite in a Brazilian teaching hospital. The American Journal of Tropical Medicine and Hygiene 47: 805-810, 1992.

20. Nunes TB, Rodrigues DS. Poisonous animals: a study of human accidents in State of Bahia, Brazil. Veterinary and Human Toxicology 29 (supl 2): 73-75, 1987.

21. Resende CC, Araújo FA, Sallenave RNVR. Análise epidemiológica dos acidentes ofídicos do Brasil - junho de 1986 a dezembro de 1987. Brasília: Ministério da Saúde. Secretaria Nacional de Ações Básicas de Saúde, 1989.

22. Ribeiro LA, Jorge MT. Acidente por serpente venenosa: dados epidemiológicos Revista da Sociedade Brasileira de Medicina Tropical 21 (supl): 121-122, 1988.

23. Ribeiro LA, Jorge MT Acidentes por serpentes do gênero Botbrops: série de 3139 casos. Revista da Sociedade Brasileira de Medicina Tropical 30: 475-480, 1997.

24. Ribeiro LA, Jorge, MT, Iversson, LB. Epidemiologia do acidentes por serpentes peçonhentas: estudo de casos atendidos em 1988. Revista de Saúde Pública 29: 380-388, 1995.

25. Rodrigues DS, Machado M.A. Poisonous animals: a study of human accidents in State of Bahia, Brazil - Part II: ophidian accidents. Veterinary and Human Toxicology 29 (supl 2): 76-78, 1987.

26. Rodrigues DS, Teles AMS, Machado MA, Vargens MM, Nascimento IM, Planzo TMP. Ofidismo na Bahia: um problema de saúde pública. Revista da Sociedade Brasileira de Toxicologia 1: 23-24, 1988

27. Rosenfeld G. Symptomatology, pathology, and treatment of snakebite in South America. In: Bücherl W, Buckley EE (eds) Venomous animals and their venoms. Academy Press, New York, volume 2, p. 345-384, 1971.

28. Santos-Costa MC. Relação antrópica e aspectos biológicos de serpentes causadoras de acidentes no Rio Grande do Sul. Comunicações do Museu de Ciências e Tecnologia da Pontifícia Universidade Católica do Rio Grande do Sul, Série Zoológica 12: 111-125, 1999.

29. Secretaria da Saúde do Estado da Bahia. Relatório Anual do Centro de Informações Antiveneno da Bahia. Governo do Estado da Bahia, Salvador, 1999.

30. Secretaria do Planejamento, Ciência e Tecnologia da Bahia. Anuário Estatístico da Bahia. SEI, Salvador, 2003.

31. Statistical Package for The Social Sciences. SPSS $®$ 11.0. SPSS Inc: Chicago, Illinois, 2001.

32. Wen FH. Soroterapia. In: Cardoso JLC, França FOS, Wen FH, Málaque CMS, Haddad V (eds) Animais peçonhentos no Brasil. Biologia, clínica e terapêutica dos acidentes. $1^{\text {a }}$ edição, Editora Sarvier, São Paulo, 468p, 2003. 